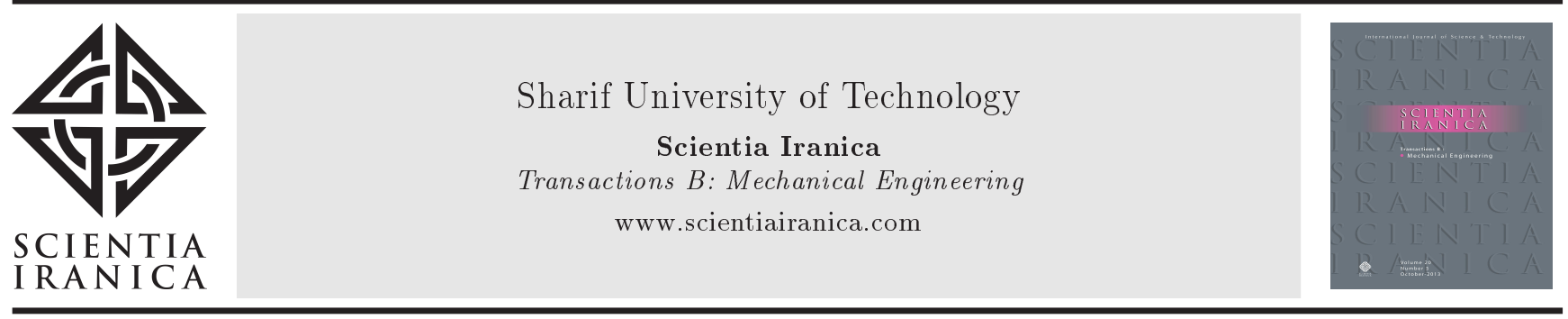

\title{
Application of homotopy perturbation method for solving nonlinear fractional heat-like equations using Sumudu transform
}

\author{
M.M. Khader ${ }^{a, b, *}$ \\ a. Department of Mathematics \& Statistics, College of Science, Al-Imam Mohammad Ibn Saud Islamic University (IMSIU), \\ Riyadh, 11566, Saudi Arabia. \\ b. Department of Mathematics, Faculty of Science, Benha University, Benha, Egypt.
}

Received 4 May 2015; received in revised form 16 February 2016; accepted 10 May 2016

\author{
KEYWORDS \\ Fractional heat-like \\ equations; \\ Caputo derivative; \\ Mittag-Leffler \\ functions; \\ Homotopy \\ perturbation method; \\ Sumudu transform \\ method.
}

\begin{abstract}
In this paper, we propose an algorithm to find approximate solutions of the proposed system of the fractional heat-like equations. The proposed algorithm, basically, illustrates how the two powerful algorithms, the Homotopy Perturbation Method (HPM) and the Sumudu Transform Method (STM), can be combined and used to get exact solutions of fractional partial differential equations. We also present some examples to illustrate the accuracy and the effectiveness of this algorithm.
\end{abstract}

(C) 2017 Sharif University of Technology. All rights reserved.

\section{Introduction}

Fractional Differential Equations (FDEs) have recently been applied to various areas of engineering, science, finance, applied mathematics, bio-engineering, and others. However, many researchers remain unaware of this field. FDEs have been the focus of many studies due to their frequent appearance in various applications in fluid mechanics, viscoelasticity, biology, physics, and engineering [1]. Consequently, considerable attention has been given to the solutions of FDEs of physical interest. Most FDEs do not have exact solutions; so, approximate and numerical techniques [2-4] must be used. Recently, several numerical methods for solving FDEs have been given, such as homotopy perturbation method [5], Adomian decomposition method [6], and collocation method [7-15].

There are numerous integral transforms such as

*. E-mail addresses: mohamed.khader@fsc.bu.edu.eg; and mohamedmbd@yahoo.com
Laplace, Sumudu, Fourier, Mellin, and Hankel to solve FDEs. Some of these, the Laplace transformation and Sumudu transformation, are most widely used. The Sumudu transformation method [16-18] is one of the most important transform methods introduced in the early 1990s by Watugala [19]. It is a powerful tool for solving many kinds of differential equations in various fields of science and engineering. And, various methods are also combined with the Sumudu transformation method, such as the homotopy analysis Sumudu transform method [20], which is a combination of the homotopy analysis method and the Sumudu transformation method. Another example is the Sumudu decomposition method [21,22], which is a combination of the Sumudu transform method and the Adomian decomposition method.

In this paper, we use the Homotopy Perturbation Sumudu Transform Method (HPSTM) to derive the exact and approximate solutions of various types of fractional partial differential equations. This method is a combination of the homotopy perturbation 
method and the Sumudu transform method. However, Singh [23] used the homotopy perturbation Sumudu transform method to obtain the exact solution of nonlinear equations which are PDEs of integer order.

Our concern in this work is to consider the numerical solution of the fractional version of the multidimensional heat-like equation. Heat-like model can describe many physical problems in different fields of science and engineering. These physical problems describe some nonlinear phenomena, such as diffusion of alleles in population genetics. The presented fractional heat-like equation has been applied in modelling to describe practical sub-diffusive problems in fluid flow process and finance [24,25].

\section{Basic definitions of fractional calculus and the Sumudu transform method}

We describe some necessary definitions and mathematical preliminaries of the fractional calculus theory and the Sumudu transform method which will be used further in this work.

Definition 1. The Caputo fractional derivative operator $D^{\alpha}$ of order $\alpha$ is defined in the following form [26]:

$$
D^{\alpha}[f(x)]=\frac{1}{\Gamma(n-\alpha)} \int_{0}^{x}(x-t)^{n-\alpha-1} \frac{d^{n} f(t)}{d t^{n}} d t .
$$

Similar to integer-order differentiation, Caputo fractional derivative operator is a linear operation:

$$
D^{\alpha}(\lambda f(x)+\mu g(x))=\lambda D^{\alpha} f(x)+\mu D^{\alpha} g(x),
$$

where $\lambda$ and $\mu$ are constants. For the Caputo's derivative, we have $D^{\alpha} C=0$, if $C$ is a constant and:

$$
D^{\alpha} x^{n}= \begin{cases}0, & \text { for } n \in \mathbb{N}_{0} \text { and } n<\lceil\alpha\rceil \\ \frac{\Gamma(n+1)}{\Gamma(n+1-\alpha)} x^{n-\alpha}, & \text { for } n \in \mathbb{N}_{0} \text { and } n \geq\lceil\alpha\rceil\end{cases}
$$

We use the ceiling function $\lceil\alpha\rceil$ to denote the smallest integer greater than or equal to $\alpha$ and $\mathbb{N}_{0}=\{0,1, \cdots\}$. Recall that for $\alpha \in \mathbb{N}$, the Caputo differential operator coincides with the usual differential operator of integer order. For more details on fractional derivatives definitions and their properties, see [27].

Definition 2. The single-parameter and the twoparameter variants of the Mittag-Leffler function are denoted by $E_{\ell}(t)$ and $E_{\ell, k}(t)$, respectively, which are relevant for their connection with fractional calculus, and are defined as:

$$
\begin{array}{ll}
E_{\ell}(t)=\sum_{j=0}^{\infty} \frac{t^{j \ell}}{\Gamma(\ell j+1)}, \quad & \ell>0, \\
E_{\ell, k}(t)=\sum_{j=0}^{\infty} \frac{t^{j \ell}}{\Gamma(\ell j+k)}, & \ell, k>0, \quad t \in C .
\end{array}
$$

Some special cases of the Mittag-Leffler function are as follows:

$$
\begin{aligned}
& E_{1}(t)=e^{t}, \\
& E_{\ell, 1}(t)=E_{\ell}(t), \\
& \frac{d^{m}}{d t^{m}}\left[t^{k-1} E_{\ell, k}\left(t^{\ell}\right)\right]=t^{k-m-1} E_{\ell, k-m}\left(t^{\ell}\right) .
\end{aligned}
$$

Other properties of the Mittag-Leffler functions can be found in [1]. These functions are generalizations of the exponential function, because most linear differential equations of fractional order have solutions that are expressed in terms of these functions.

Definition 3. The Sumudu transform is defined over the set of functions:

$$
\begin{aligned}
A= & \left\{f(t)\left|\exists M, \tau_{1}, \tau_{2}>0,\right| f(t) \mid<M e^{|t| / \tau_{j}},\right. \\
& \text { if } \left.t \in(-1)^{j} \times[0, \infty)\right\},
\end{aligned}
$$

by the following formula:

$$
F(u)=S[f(t)]=\int_{0}^{\infty} f(u t) e^{-t} d t, \quad u \in\left(-\tau_{1}, \tau_{2}\right) .
$$

Some special properties of the Sumudu transform (denoted throughout this paper by $S$ ) are as follows:

1. $S[1]=1$;

2. $S\left[\frac{t^{n}}{\Gamma(n+1)}\right]=u^{n}, n>0$;

3. $S\left[e^{a t}\right]=\frac{1}{1-a u}$;

4. $S[\lambda f(t)+\mu g(t)]=\lambda S[f(t)]+\mu S[g(t)]$.

Other properties of the Sumudu transform can be found in [21].

Definition 4. The Sumudu transform, $S\left[D^{\alpha} f(t)\right]$ of the Caputo fractional derivative, is defined as [20]:

$$
S\left[D^{\alpha} f(t)\right]=\frac{F(u)}{u^{\alpha}}-\sum_{k=0}^{n-1} \frac{f^{(k)}(0)}{u^{\alpha-k}}, \quad n-1<\alpha \leq n,
$$

where $F(u)$ is the Sumudu transform of $f(t)$. Then, it can be easily understood that:

$$
S\left[D_{t}^{\alpha} f(x, t)\right]=\frac{S[f(x, t)]}{u^{\alpha}}-\sum_{k=0}^{n-1} \frac{f^{(k)}(x, 0)}{u^{\alpha-k}} .
$$




\section{The basic idea of HPM}

In this section, we will briefly present the algorithm of HPM. At first, we consider the following nonlinear differential equation:

$$
A(u)-f(r)=0, \quad r \in \Omega,
$$

with the boundary conditions:

$$
B\left(u, \frac{\partial u}{\partial n}\right)=0, \quad r \in \Gamma
$$

where $A, B, f(r)$, and $\Gamma$ are the general differential function operator, the boundary operator, the known analytical function, and the boundary of the domain $\Omega$, respectively.

The operator $A$ can be decomposed into a linear operator denoted by $L$, and a nonlinear operator denoted by $N$. Therefore, Eq. (3) can be written as follows:

$$
L(u)+N(u)-f(r)=0 .
$$

Now, we construct a homotopy $v(r, p): \Omega \times[0,1] \rightarrow \Re$ which satisfies:

$$
\begin{aligned}
H(v, p) & =(1-p)\left[L(v)-L\left(u_{0}\right)\right]+p[A(v)-f(r)]=0, \\
0 \leq p & \leq 1,
\end{aligned}
$$

which is equivalent to:

$$
\begin{aligned}
& H(v, p)=L(v)-L\left(u_{0}\right)+p L\left(u_{0}\right)+p[N(v)-f(r)]=0, \\
& 0 \leq p \leq 1,
\end{aligned}
$$

where $u_{0}$ is the initial approximation of Eq. (3) that satisfies the boundary conditions (4), and $p$ is an embedding parameter.

When the value of $p$ is changed from zero to unity, we can easily see that:

$$
\begin{aligned}
H(v, 0)= & L(v)-L\left(u_{0}\right)=0, \\
H(v, 1)= & L(v)+N(v)-f(r)=A(v) \\
& -f(r)=0 .
\end{aligned}
$$

In topology, this changing process is called deformation, and Eqs. (8) and (9) are called homotopic. If the $p$-parameter is considered as small, then the solution of Eq. (5) can be expressed as a power series in $p$ :

$$
\begin{aligned}
& v=v_{0}+p v_{1}+p^{2} v_{2}+p^{3} v_{3}+\cdots, \\
& u=\lim _{p \rightarrow 1} v=v_{0}+v_{1}+v_{2}+v_{3}+\cdots
\end{aligned}
$$

\section{The homotopy perturbation Sumudu transform method}

In order to elucidate the solution procedure of this method, we consider the general fractional nonlinear partial differential equation of the form [20]:

$$
\begin{gathered}
D_{t}^{\alpha} w(r, t)=L w(r, t)+N w(r, t)+q(r, t), \\
n-1<\alpha \leq n,
\end{gathered}
$$

subject to the initial conditions:

$$
w^{(m)}(r, 0)=f_{m}(r), \quad m=0,1, \cdots, n-1,
$$

where $D_{t}^{\alpha} w(r, t)$ is the Caputo fractional derivative with respect to $t, q(r, t)$ is the source term, $L$ is the linear operator, and $N$ is the general nonlinear operator.

Taking the Sumudu transform on both sides of Eq. (12), we get:

$$
S\left[D_{t}^{\alpha} w(r, t)\right]=S[L w(r, t)+N w(r, t)+q(r, t)] .
$$

Using the property of the Sumudu transform and the initial conditions in Eq. (13), we have:

$$
\begin{array}{r}
u^{-\alpha} S[w(r, t)]-\sum_{k=0}^{n-1} u^{-(\alpha-k)} w^{(k)}(r, 0) \\
=S[L w(r, t)+N w(r, t)+q(r, t)],
\end{array}
$$

and:

$$
\begin{aligned}
S[w(r, t)]= & \sum_{k=0}^{n-1} u^{k} f_{k}(r)+u^{\alpha} S[L w(r, t) \\
& +N w(r, t)+q(r, t)] .
\end{aligned}
$$

Operating with the Sumudu inverse on both sides of Eq. (15), we get:

$$
\begin{aligned}
w(r, t)= & S^{-1}\left[\sum_{k=0}^{n-1} u^{k} f_{k}(r)\right]+S^{-1}\left[u^{\alpha} S[L w(r, t)\right. \\
& +N w(r, t)+q(r, t)]] .
\end{aligned}
$$

Now, by applying the classical perturbation technique, we can find the solution of Eq. 16) in the form:

$$
w(r, t)=\sum_{k=0}^{\infty} p^{k} w_{k}(r, t),
$$

where $p \in[0,1]$ is the homotopy parameter. The nonlinear term of Eq. (16) can be decomposed as:

$$
N w(r, t)=\sum_{k=0}^{\infty} p^{k} H_{k}(w),
$$


where $H_{k}$ is He's polynomial, which can be calculated with the formulas $[6,20]$ as follows:

$$
\begin{aligned}
& H_{k}\left(w_{0}, w_{1}, w_{2}, \cdots, w_{n}\right)=\frac{1}{k !} \frac{\partial^{k}}{\partial p^{k}}\left[N\left(\sum_{i=0}^{\infty} p^{i} w_{i}\right)\right]_{p=0}, \\
& k=0,1, \cdots
\end{aligned}
$$

Substituting Eqs. (17) and (18) into Eq. (16), we get:

$$
\begin{gathered}
\sum_{k=0}^{\infty} p^{k} w_{k}(r, t)=S^{-1}\left[\sum_{k=0}^{n-1} u^{k} f_{k}(r)\right] \\
+p S^{-1}\left[u ^ { \alpha } S \left[L\left(\sum_{k=0}^{\infty} p^{k} w_{k}(r, t)\right)\right.\right. \\
\left.\left.+\sum_{k=0}^{\infty} p^{k} H_{k}(w)+q(r, t)\right]\right]
\end{gathered}
$$

Equating the terms with identical powers of $p$, we can obtain a series of equations as follows:

$$
\begin{aligned}
p^{0}: \quad w_{0}(r, t)= & S^{-1}\left[\sum_{k=0}^{n-1} u^{k} f_{k}(r)\right], \\
p^{1}: \quad w_{1}(r, t)= & S^{-1}\left[u ^ { \alpha } S \left[L w_{0}(r, t)\right.\right. \\
& \left.\left.+H_{0}(w)+q(r, t)\right]\right], \\
p^{2}: \quad w_{2}(r, t)= & S^{-1}\left[u ^ { \alpha } S \left[L w_{1}(r, t)\right.\right. \\
& \left.\left.+H_{1}(w)+q(r, t)\right]\right], \cdots, \\
p^{n}: \quad w_{n}(r, t)= & S^{-1}\left[u ^ { \alpha } S \left[L w_{n-1}(r, t)\right.\right. \\
& \left.\left.+H_{n-1}(w)+q(r, t)\right]\right] .
\end{aligned}
$$

By utilizing the results in Eq. (21) and substituting them into Eq. (17), the solution of Eq. (12) can be expressed as a power series in $p$. The best approximation for the solution of Eq. (12) is:

$$
w(r, t)=\lim _{p \rightarrow 1} \sum_{k=0}^{\infty} p^{k} w_{k}(r, t)=w_{0}+w_{1}+w_{2}+\cdots
$$

The solutions in Eq. (22) generally converge very rapidly [20].

\section{Illustrative examples}

In this section, in order to assess the applicability and the accuracy of the fractional homotopy perturbation Sumudu transform method in the last section, we consider the following two examples.
Example 1. Consider the following fractional twodimensional heat-like equation of the form:

$$
D_{t}^{\alpha} w(x, y, t)=\frac{1}{2}\left(y^{2} w_{x x}+x^{2} w_{y y}\right), \quad 0<\alpha \leq 1,
$$

subject to the initial condition:

$$
w(x, y, 0)=y^{2}
$$

subject to the boundary conditions:

$$
\begin{aligned}
w_{x}(0, y, t) & =0, & w_{y}(x, 0, t) & =0, \\
w_{x}(1, y, t) & =2 \sinh t, & w_{y}(x, 1, t) & =2 \cosh t .
\end{aligned}
$$

Taking the Sumudu transform on both sides of Eq. (23), we get:

$$
S\left[D_{t}^{\alpha} w(x, y, t)\right]=S\left[\frac{1}{2}\left(y^{2} w_{x x}+x^{2} w_{y y}\right)\right] .
$$

Using the property of the Sumudu transform and the initial condition in Eq. (24), we have:

$$
S[w(x, y, t)]=w(x, y, 0)+u^{\alpha} S\left[\frac{1}{2}\left(y^{2} w_{x x}+x^{2} w_{y y}\right)\right] \text {. }
$$

Operating with the inverse of Sumudu on both sides of Eq. (26), we get:

$$
w(x, y, t)=S^{-1}\left[y^{2}\right]+S^{-1}\left[\frac{1}{2} u^{\alpha} S\left[y^{2} w_{x x}+x^{2} w_{y y}\right]\right]
$$

By applying the homotopy perturbation method and substituting Eq. (17) into Eq. (27), we have:

$$
\begin{aligned}
& \sum_{k=0}^{\infty} p^{k} w_{k}(x, y, t)=y^{2} \\
& +p S^{-1}\left[\frac { 1 } { 2 } u ^ { \alpha } S \left[y^{2}\left(\sum_{k=0}^{\infty} p^{k} w_{k x x}(x, y, t)\right)\right.\right. \\
& \left.\left.+x^{2}\left(\sum_{k=0}^{\infty} p^{k} w_{k y y}(x, y, t)\right)\right]\right] .
\end{aligned}
$$

Equating the terms with identical powers of $p$, we get:

$$
\begin{array}{ll}
p^{0}: & w_{0}(x, y, t)=y^{2} \\
p^{1}: & w_{1}(x, y, t)=x^{2} \frac{t^{\alpha}}{\Gamma(\alpha+1)}, \\
p^{2}: & w_{2}(x, y, t)=y^{2} \frac{t^{2 \alpha}}{\Gamma(2 \alpha+1)}, \\
p^{3}: & w_{3}(x, y, t)=x^{2} \frac{t^{3 \alpha}}{\Gamma(3 \alpha+1)}, \\
p^{4}: & w_{4}(x, y, t)=y^{2} \frac{t^{4 \alpha}}{\Gamma(4 \alpha+1)}, \\
p^{5}: & w_{5}(x, y, t)=x^{2} \frac{t^{5 \alpha}}{\Gamma(5 \alpha+1)}, \cdots .
\end{array}
$$


Thus, the approximate solution of Eq. (23) is given by:

$$
\begin{aligned}
& w(x, y, t)=\lim _{p \rightarrow 1} \sum_{k=0}^{\infty} p^{k} w_{k}(x, y, t) \\
& =x^{2}\left[\frac{t^{\alpha}}{\Gamma(\alpha+1)}+\frac{t^{3 \alpha}}{\Gamma(3 \alpha+1)}+\cdots\right] \\
& +y^{2}\left[1+\frac{t^{2 \alpha}}{\Gamma(2 \alpha+1)}+\frac{t^{4 \alpha}}{\Gamma(4 \alpha+1)}+\cdots\right] \\
& =x^{2} \sum_{k=0}^{\alpha} \frac{t^{(2 k+1) \alpha}}{\Gamma((2 k+1) \alpha+1)}+y^{2} \sum_{k=0}^{\alpha} \frac{t^{2 k \alpha}}{\Gamma(2 k \alpha+1)} \\
& =x^{2} \sinh \left(t^{\alpha}, \alpha\right)+y^{2} \cosh \left(t^{\alpha}, \alpha\right),
\end{aligned}
$$

where $\sinh (\ell, \alpha)$ and $\cosh (\ell, \alpha)$ are defined as follows:

$$
\begin{aligned}
& \sinh (\ell, \alpha)=\frac{E_{\alpha}(\ell)-E_{\alpha}(-\ell)}{2}, \\
& \cosh (\ell, \alpha)=\frac{E_{\alpha}(\ell)+E_{\alpha}(-\ell)}{2} .
\end{aligned}
$$

If we put $\alpha \rightarrow 1$ in Eq. (30), we obtain the exact solution of Eq. (23).

$$
w(x, y, t)=x^{2} \sinh (t)+y^{2} \cosh (t) .
$$

The results for the exact solution of Eq. (31) and the approximate solution of Eq. (30) obtained using HPSTM for the special case of $\alpha=1$, at $x=y=$ $1.5, t \in[0,2]$, are shown in Figure 1. From this figure, it can be seen that the solution obtained by the presented method is nearly identical to the exact solution. Figure 2 shows the approximate solution with different values of $\alpha(\alpha=0.7,0.8,0.9,1)$ at $x=$ $y=1, t \in[0,2]$. It is to be noted that only the five-order term of the solution was used in evaluating

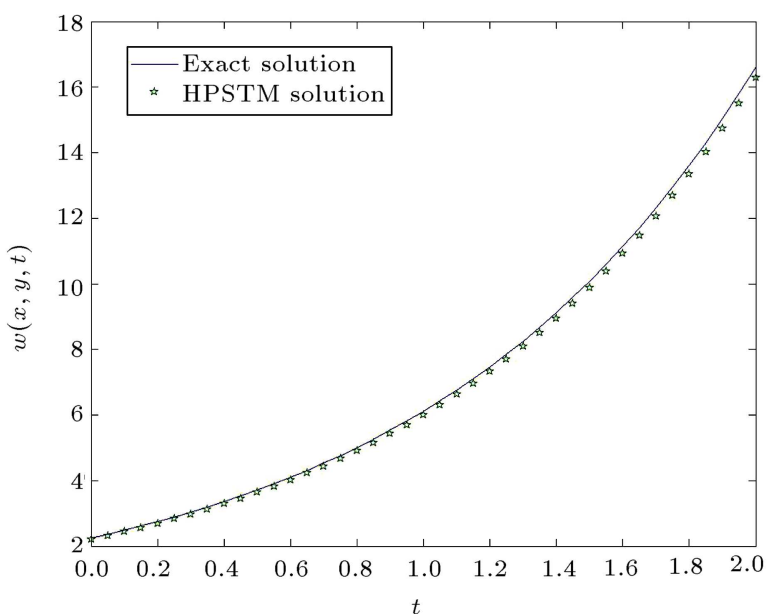

Figure 1. The behavior of the approximate solution and the exact solution with $\alpha=1$, at $x=y=1.5$.

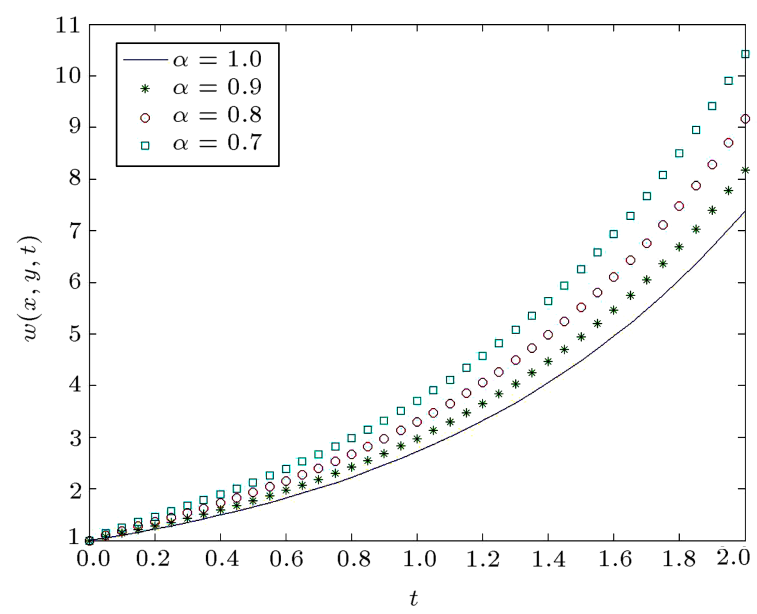

Figure 2. The behavior of the approximate solution of Example 1 with different values of $\alpha$.

the approximate solutions for Figure 2. It is evident that the efficiency of this approach can be dramatically enhanced by computing further terms of $w(x, y, t)$ when the homotopy perturbation Sumudu transform method is used.

Example 2. Consider the following fractional threedimensional heat-like equation of the form:

$$
\begin{aligned}
& D_{t}^{\alpha} w(x, y, z, t)=x^{4} y^{4} z^{4} \\
& \quad+\frac{1}{36}\left(x^{2} w_{x x}+y^{2} w_{y y}+z^{2} w_{z z}\right), \quad 0<\alpha \leq 1,
\end{aligned}
$$

subject to the initial condition:

$$
w(x, y, z, 0)=0,
$$

subject to Neumann boundary conditions:

$$
\begin{aligned}
& w(0, y, z, t)=w(x, 0, z, t)=w(x, y, 0, t)=0, \\
& w(1, y, z, t)=y^{4} z^{4}\left(e^{t}-1\right), \\
& w(x, 1, z, t)=x^{4} z^{4}\left(e^{t}-1\right), \\
& w(x, y, 1, t)=x^{4} y^{4}\left(e^{t}-1\right) .
\end{aligned}
$$

Taking the Sumudu transform on both sides of Eq. (32), we get:

$$
\begin{aligned}
S & {\left[D_{t}^{\alpha} w(x, y, z, t)\right] } \\
& =S\left[x^{4} y^{4} z^{4}+\frac{1}{36}\left(x^{2} w_{x x}+y^{2} w_{y y}+z^{2} w_{z z}\right)\right] .
\end{aligned}
$$

Using the property of the Sumudu transform and the initial condition in Eq. (33), we have: 


$$
\begin{aligned}
& S[w(x, y, z, t)]=u^{\alpha} S\left[x^{4} y^{4} z^{4}\right] \\
& \quad+u^{\alpha} S\left[\frac{1}{36}\left(x^{2} w_{x x}+y^{2} w_{y y}+z^{2} w_{z z}\right)\right] .
\end{aligned}
$$

Operating with the inverse of Sumudu on both sides of Eq. (35), we get:

$$
\begin{aligned}
& w(x, y, z, t)=S^{-1}\left[u ^ { \alpha } S \left[x^{4} y^{4} z^{4}\right.\right. \\
& \left.\left.+\frac{1}{36}\left(x^{2} w_{x x}+y^{2} w_{y y}+z^{2} w_{z z}\right)\right]\right] .
\end{aligned}
$$

By applying the homotopy perturbation method and substituting Eq. (17) into Eq. (36), we have:

$$
\begin{aligned}
& \sum_{k=0}^{\infty} p^{k} w_{k}(x, y, z, t)=p S^{-1}\left[u^{\alpha} S\left[x^{4} y^{4} z^{4}\right]\right. \\
& +\frac{1}{36} u^{\alpha} S\left[x^{2}\left(\sum_{k=0}^{\infty} p^{k} w_{k x x}(x, y, z, t)\right)\right. \\
& +y^{2}\left(\sum_{k=0}^{\infty} p^{k} w_{k y y}(x, y, z, t)\right) \\
& \left.\left.\quad+z^{2}\left(\sum_{k=0}^{\infty} p^{k} w_{k z z}(x, y, z, t)\right)\right]\right] .
\end{aligned}
$$

Equating the terms with identical powers of $p$, we get:

$$
\begin{array}{ll}
p^{0}: & w_{0}(x, y, z, t)=0, \\
p^{1}: & w_{1}(x, y, z, t)=\frac{x^{4} y^{4} z^{4} t^{\alpha}}{\Gamma(\alpha+1)}, \\
p^{2}: & w_{2}(x, y, z, t)=\frac{x^{4} y^{4} z^{4} t^{2 \alpha}}{\Gamma(2 \alpha+1)}, \cdots, \\
p^{n}: & w_{n}(x, y, z, t)=\frac{x^{4} y^{4} z^{4} t^{n \alpha}}{\Gamma(n \alpha+1)} .
\end{array}
$$

Thus, the approximate solution of Eq. (32) is given by:

$$
\begin{aligned}
w(x, y, z, t) & =\lim _{p \rightarrow 1} \sum_{k=0}^{\infty} p^{k} w_{k}(x, y, z, t) \\
& =\left(x^{4} y^{4} z^{4}\right)\left[E_{\alpha}\left(t^{\alpha}\right)-1\right] .
\end{aligned}
$$

If we put $\alpha \rightarrow 1$ in Eq. (39) or solve Eqs. (32) and (33) with $\alpha=1$, we obtain the exact solution:

$$
w(x, y, z, t)=x^{4} y^{4} z^{4}\left(e^{t}-1\right) .
$$

The results for the exact solution of Eq. (40) and the approximate solution of Eq. (39) obtained using

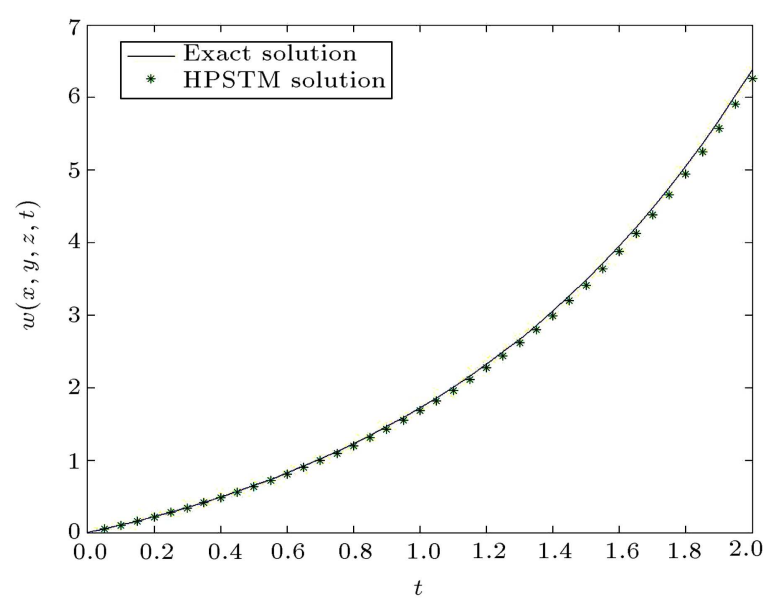

Figure 3. The behavior of the approximate solution and the exact solution with $\alpha=1$, at $x=y=z=1.0$.

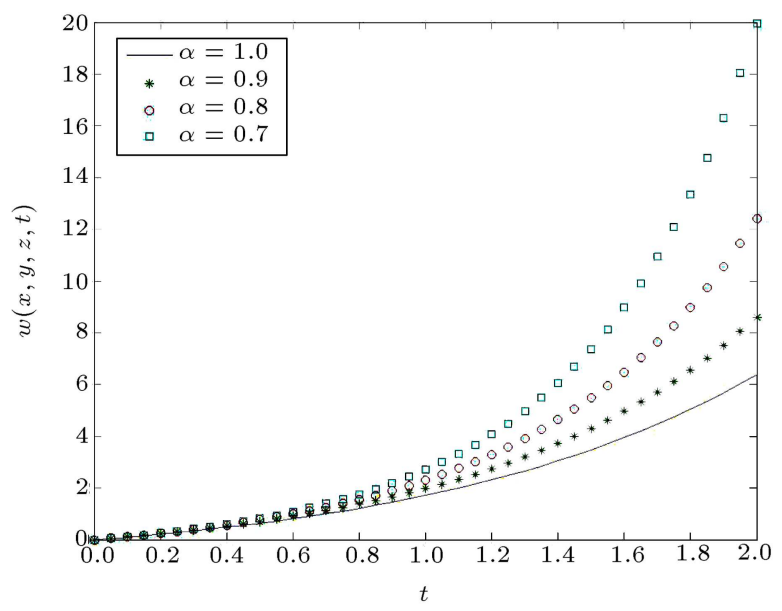

Figure 4. The behavior of the approximate solution of example 2 with different values of $\alpha$.

HPSTM for the special case of $\alpha=1$, at $x=y=$ $z=1.0, t \in[0,2]$, are shown in Figure 3. From this figure, it can be seen that the solution obtained by the presented method is nearly identical to the exact solution. Figure 4 shows the approximate solution with different values of $\alpha(\alpha=0.7,0.8,0.9,1)$ at $x=y=$ $z=1.0, t \in[0,2]$. It is to be noted that only the five-order term of the solution was used in evaluating the approximate solutions for Figure 3. It is evident that the efficiency of this approach can be dramatically enhanced by computing further terms of $w(x, y, z, t)$ when the homotopy perturbation Sumudu transform method is used. In addition, from all presented Figures 1-4, we can confirm that the behavior of the numerical solution has satisfied the physical meaning of the problem under consideration.

\section{Conclusion and remarks}

In this article, we implemented the homotopy perturbation Sumudu transform method to obtain the 
approximate solutions of the multi-dimensional fractional heat-like equations. However, HPSTM has an advantage over SDM, that is, it solves the nonlinear problems without using Adomian's polynomials. In conclusion, HPSTM may be considered as a nice refinement in existing numerical technique and might find the wide applications. The obtained approximate solution using the suggested method is in excellent agreement with the exact solution, and shows that these approaches can solve the problem effectively and illustrate the validity and the great potential of the proposed technique. All computations in this paper are done using Matlab 8.

\section{References}

1. Podlubny, I., Fractional Differential Equations, Academic Press, New York (1999).

2. He, J.H. "A tutorial review on fractal space-time and fractional calculus", International Journal of Theoretical Physics, 53(11), pp. 3698-3718 (2014).

3. Sweilam, N.H. and Khader, M.M. "A Chebyshev pseudo-spectral method for solving fractional integrodifferential equations", ANZIAM Journal, 51, pp. 464475 (2010).

4. Sweilam, N.H., Khader, M.M. and Nagy, A.M. "Numerical solution of two-sided space-fractional wave equation using finite difference method", J. of Computional and Applied Mathematics, 235, pp. 2832-2841 (2011).

5. Ghorbani, A. and Saberi-Nadjafi, J. "He's homotopy perturbation method for calculating Adomian's polynomials", Inter. J. of Nonlinear Sciences and Numerical Simulation, 8, pp. 229-232 (2007).

6. Ghorbani, A. "Beyond Adomian's polynomials: He's polynomials", Chaos Solitons and Fractals, 39, pp. 1486-1492 (2009).

7. Khader, M.M. "On the numerical solutions for the fractional diffusion equation", Communications in Nonlinear Science and Numerical Simulations, 16, pp. 25352542 (2011).

8. Khader, M.M. "An efficient approximate method for solving linear fractional Klein-Gordon equation based on the generalized Laguerre polynomials", International Journal of Computer Mathematics, 90(9), pp. 1853-1864 (2013).

9. Khader, M.M. "An efficient approximate method for solving fractional variational problems", Applied Mathematical Modelling, 39, pp. 1643-1649 (2015).

10. Khader, M.M. and Sweilam, N.H. "On the approximate solutions for system of fractional integrodifferential equations using Chebyshev pseudo-spectral method", Applied Mathematical Modelling, 37, pp. 9819-9828 (2013).

11. Khader, M.M. and Hendy, A.S. "A numerical tech- nique for solving fractional variational problems", Mathematical Methods in Applied Sciences, 36(10), pp. 1281-1289 (2013).

12. Khader, M.M., Sweilam, N.H. and Mahdy, A.M.S. "Numerical study for the fractional differential equations generated by optimization problem using Chebyshev collocation method and FDM", Applied Mathematics and Information Science, 7(5), pp. 2013-2020 (2013).

13. Khader, M.M. and Adel, M. "Numerical solutions of fractional wave equations using an efficient class of FDM based on Hermite formula", Advances in Difference Equations, 2016(34), pp. 1-10 (2016).

14. Khader, M.M., Sweilam, N.H., Mahdy, A.M.S. and Abdel Moniem, N.K. "Numerical simulation for the fractional SIRC model and influenza A", Applied Mathematics and Information Science, 8(3), pp. 1029-1036 (2014).

15. Sweilam, N.H., Khader, M.M. and Mahdy, A.M.S. "Numerical studies for fractional-order Logistic differential equation with two different delays", Journal of Applied Mathematics, 2012, pp. 1-14 (2012).

16. Atangana, A. and Adem, K. "The use of Sumudu transform for solving certain nonlinear fractional heatlike equations", Abstract and Applied Analysis, 2013, pp. 1-12 (2013).

17. Aydin, S. "Approximate analytic solution of fractional heat-like and wave-like equations with variable coefficients using the differential transforms method", Advances in Difference Equations, 2012(198), pp. 19 (2012).

18. Das, S. "A note on fractional diffusion equations", Chaos, Solitons and Fractals, 42, pp. 2074-2079 (2009).

19. Watugala, G.K. "Sumudu transform -a new integral transform to solve differential equations and control engineering problems", Math. Engg. Indust., 6(4), pp. 319-329 (1998).

20. Karbalaie, A., Montazeri, M.M. and Muhammed, H.H. "Exact solution of time-fractional partial differential equations using Sumudu transform", WSEAS Transactions on Mathematics, 13, pp. 142-151 (2014).

21. Belgacem, F.B.M. and Karaballi, A.A. "Sumudu transform fundamental properties investigations and applications", Inter. J. Appl. Math. Stoch. Anal., 2006, pp. 1-23 (2005).

22. Kumar, D., Singh, J. and Sushila, "Sumudu decomposition method for nonlinear equations", International Mathematical Forum, 7(11), pp. 515-521 (2012).

23. Singh, B.K. and Srivastava, V.K. "Approximate series solution of multi-dimensional, time fractional-order (heat-like) diffusion equations using FRDTM", Royal Society Open Science, 2(4), pp. 140511-140524 (2015).

24. Jamshad, A., Iftikhar, A. and Bilal, A. "Solutions of 
fractional heat-and wave-like equations via fractional variational iteration method", Journal of Mathematical Sciences: Advances and Applications, 21, pp. 1-15 (2013).

25. Yousif, E.A. and Hamed, S.H.M. "Solution of nonlinear fractional differential equations using the homotopy perturbation Sumudu transform method", Applied Mathematical Sciences, 8(44), pp. 2195-2210 (2014).

26. Liu, F.J., Li, Z.B., Zhang, S. and Liu, H.Y. "He's fractional derivative for heat conduction in a fractal medium arising in Silkworm Cocoon hierarchy", Thermal Science, 19(4), pp. 1155-1159 (2015).
27. Oldham, K.B. and Spanier, J., The Fractional Calculus, Academic Press, New York (1974).

\section{Biography}

Mohamed Meabed Khader was born in 1973 in Egypt. He received his $\mathrm{BS}, \mathrm{MS}$, and $\mathrm{PhD}$ degrees from Benha University, Egypt, in 1995, 2001, and 2009, respectively, and is currently an Associate Professor in the Mathematical Department of IMAM University, KSA. His main research interests are the Numerical Analysis and Computational Fluid. 